\title{
Modulation of chondrocyte phenotype for tissue engineering by designing the biologic-polymer carrier interface
}

\author{
Tahir A. Mahmood, ${ }^{1,2^{*}}$ Sylvie Miot, ${ }^{3}$ Oliver Frank, ${ }^{3}$ Ivan Martin, ${ }^{3}$ Jens Riesle, ${ }^{1}$ \\ Robert Langer ${ }^{2}$ and Clemens A. van Blitterswijk ${ }^{1}$
}

${ }^{1}$ Institute of Biomedical Technology, University of Twente, Prof. Bronkhorstlaan 10, 3723 MB Bilthoven, The Netherlands

${ }^{2}$ Department of Chemical Engineering, Massachusetts Institute of Technology, 45 Carleton St. E25-342, Cambridge, Massachusetts 02139, United States

${ }^{3}$ Division of Research, University of Basel Hospital, Hebelstrasse 20, Basel 4031, Switzerland

\footnotetext{
* Current address of corresponding author:

Tahir A. Mahmood, Ph.D.

Department of Pharmaceutics

Amgen

One Amgen Center Drive

$\mathrm{M} / \mathrm{S}$ 8-2-D

Thousand Oaks, CA 91320

United States

Phone 1.805.447.1000

Email tmahmood@amgen.com
} 


\section{ABSTRACT}

Therapeutic strategies based on cell and tissue engineering can be advanced by developing material substrates that effectively interrogate the biological compartment, with or without, the complimentary local release of growth factors. Poly(ether ester) segmented copolymers were engineered as model material systems to elucidate the interfacial molecular events that govern the function of adhered cells. Surface chemistry was modulated by varying poly(ethylene glycol) (PEG) length and mole fraction with poly(butylene terephthalate) (PBT), leading to differential competitive protein adsorption of fibronectin and vitronectin from serum, and consequently to different cell attachment modes. Adhesion within the hydrogellike milieu of longer surface PEG was mediated via binding to the CD44 transmembrane receptor, rather than the RGD-integrin mechanism, whereas greater substrate-bound fibronectin resulted in cell adhesion via integrins. These adhesion modalities differentially impacted morphological cell phenotype (spread or spheroid) and the subsequent expression

of mRNA transcripts (collagen types II, I) characteristic of phenotypically differentiated or dedifferentiated chondrocytes, respectively. These results demonstrate that materials can be designed to directly elicit the membrane bound receptor apparatus desired for downstream cellular response, without requiring exogenous biological growth factors to enable differentiated potential.

Keywords: copolymer, PEG, cell transplantation, tissue engineering, chondrocyte, gene expression, protein adsorption, dedifferentiation, adhesion, growth factors. 


\section{INTRODUCTION}

Clinical bioengineering therapies, such as cell and tissue engineering, can involve the use of material substrates as delivery systems by which to transplant cells to diseased, damaged or resected tissue. Once placed into the patient, hybrid material-biologic constructs allow the transplanted cells, or induce cells at the peri-implant site, to produce tissue extracellular matrix $(E C M)$ or endogenous growth factors required for tissue regeneration or repair ${ }^{1}$. Strategies employed to achieve the desired cellular response include the use of exogenous growth factors to enable differentiated potential ${ }^{2}$, as well as incorporation of peptide ligands or growth factors within the delivery substrate to induce bioactive functionality ${ }^{3}$. However, little is understood regarding the mechanistic interfacial events that have the potential to regulate cell function without the use of exogenous bioactive growth factors or coatings.

For the development of such composite material-cellular technologies, it is necessary to understand the interaction between the material surface and the cellular/biological compartment, from protein adsorption to cell adhesion and downstream intracellular events. Cell-material interaction was first studied to understand the cultivation of anchorage dependent mammalian cells on culture substrates $^{4-6}$, and more recently for cell delivery applications $^{7-9}$. Several substrate properties have been proposed as potential regulators of cell function, including surface wettability, flexibility and roughness ${ }^{10}$. In serum containing culture conditions, however, cells would be expected to 'sense' the biochemical environment including proteins adsorbed at the surface, and not simply the native material chemistry. It has been suggested that a combination of substrate parameters collectively influence cell function by modulating the adsorption of proteins, with the resulting proteinaceous milieu at the surface impacting cell function ${ }^{11,12}$. 
A major challenge in current chondrocyte cell therapy arises from interactions between the cells and their delivery substrates. Chondrocyte attachment and spreading on surfaces is known to lead to 'dedifferentiation' to a more fibroblast-like cell type, i.e. loss of hyaline cartilage ECM protein synthesis (Type II collagen and aggrecan), and the upregulation of Type I collagen synthesis ${ }^{13-17}$. In addition, it has been shown that the presence of fibronectin $(\mathrm{Fn})$ can induce chondrocyte dedifferentiation in monolayer culture ${ }^{18,19}$. Vitronectin $(\mathrm{Vn})$ has also shown to be involved in chondrocyte adhesion to some synthetic polymer substrates ${ }^{20}$.

Segmented poly(ether ester) copolymers of poly(ethylene glycol)-terephthalate (PEGT) and poly(butylene terephthalate) (PBT) (PEGT:PBT) were synthesized as model substrates for studying material-directed cell function and cell transplantation, due to the flexibility in design afforded by the constituent polymers. This polymer system is also currently under evaluation as a substrate for tissue engineering applications. Copolymer properties are determined by the two components - the PEG segments contain mobile hydrophilic chains to provide a hydrogel-like local environment, whereas PBT provide hydrophobic, protein-binding domains and rigidity to the hybrid system ${ }^{21}$ (Figure 1). During synthesis, the molecular mass of PEG and weight ratio of the PEGT:PBT components can be tailored to endow polymer substrates with specific surface and mechanical properties, as dictated by individual cell delivery applications.

The objective of this study was to elucidate the effects of varying model poly(ether ester) copolymer substrate parameters on the differential regulation of cell phenotype, and to identify interactions between materials, proteins and cells at the interfacial level that contribute to material-based cell regulation without the use of biological growth factors or coatings. 


\section{METHODS}

\section{Polymer preparation}

Synthesis: PEGT:PBT segmented copolymers were prepared by two-step condensation in the presence of titanium tetrabutoxide (Merck; Darmstadt, Germany) as catalyst $(0.1 \mathrm{wt} \%)^{21}$. Vitamin E (Sigma; Uithoorn, The Netherlands) was included as an anti-oxidant for the polymers. Compositions were varied by changing PEG molecular mass, and PEG to dimethyl terephthalate/1, 4 butanediol (Merck) ratio.

Polymer nomenclature: The different formulations of this copolymer system are indicated as: $a$-PEG $b: c$, where $a$ is the molecular mass of PEG $(\mathrm{g} / \mathrm{mole}), b$ is the mass percentage of PEGT and $c$ is the mass percentage of the PBT component. For example, the copolymer 300-PEG 55:45 has PEG molecular mass of $300 \mathrm{~g} / \mathrm{mole}$ and a PEGT:PBT ratio of 55:45. Tissue culture polystyrene (TCPS) was included as a control surface. A list of polymer compositions engineered for this study per the nomenclature used is provided in Table1.

Substrate preparation: $20 \%(\mathrm{w} / \mathrm{v})$ polymer solutions in either chloroform (Sigma) or in a mixture of chloroform and 1,1,1,3,3,3-hexafluoro-2-propanol (Sigma) were cast into twodimensional (2D) polymer substrate films $(60-100 \mu \mathrm{m}$ thick) on glass. The polymer substrates were placed in ethanol (12 hours) to remove residual solvent, vacuum dried under $\mathrm{N}_{2}$ (48 hours), $\gamma$-sterilized and immersed in serum-containing culture medium (12 hours) prior to cell seeding. The wettabilities of individual polymer compositions have been tested and reported previously 22,23 . 
Tissue harvesting and cell isolation

Non-osteoarthritic articular cartilage was harvested from the femoral heads of female patients undergoing hip replacement surgery, under institutional standards of informed consent at the University Hospital of Basel. A representative set of complete data from one 65-year old female patient was selected. Primary chondrocytes were isolated by type II collagenase (Worthington Biochemical, Lakewood, NJ) digestion for 20 hours, rinsed in phosphate buffered saline (PBS) containing $\mathrm{CaCl}_{2}$ and $\mathrm{MgCl}_{2}$ at $\mathrm{pH} 7.4$ (Invitrogen; Breda, The Netherlands) with $15 \%$ fetal bovine serum (FBS) (Invitrogen) and transferred to a well defined culture medium (Dulbecco's Modified Eagle Medium (DMEM) containing $4.5 \mathrm{~g} / \mathrm{l}$ glucose and supplemented with 10\% FBS, $1 \mathrm{mM}$ sodium pyruvate (Invitrogen), 50 $\mu \mathrm{g} / \mathrm{ml}$ penicillin, $50 \mu \mathrm{g} / \mathrm{ml}$ streptomycin, $0.4 \mathrm{mM}$ L-proline, $0.1 \mathrm{mM}$ non-essential amino acids and $10 \mathrm{mM}$ Hepes buffer (Sigma)).

Once isolated, cells were seeded at a density of 10,000 cells $/ \mathrm{cm}^{2}$ on each polymer. Attached cells were quantitated as described below at 1,13 and 19 days after seeding, assessed morphologically and for phenotypic mRNA transcript expression at day 19. All samples were evaluated in duplicate. The limited sample sizes were due to difficulty in obtaining sufficient grossly non-osteoarthritic human cartilage from one biopsy, which was necessary for a consistent baseline cellular phenotype.

Real time quantitative polymerase chain reaction (RT-PCR)

Samples were frozen in Trizol reagent (Sigma) at $-80^{\circ} \mathrm{C}$ after harvesting at day 19. RNA were extracted using Trizol. cDNA were generated by using Stratascript reverse transcriptase (Stratagene, Amsterdam, The Netherlands) in the presence of dNTP according to the manufacturer's instructions. PCR reactions were performed and monitored using a $A B I$ 
Prism 7700 sequence Detection System (Perkin-Elmer Applied Biosystems). The PCR master mix was based on AmpliTaq Gold DNA polymerase (Perkin-Elmer Appplied Biosystems). cDNA samples were analyzed in duplicates. Sequences of primers and probes for human collagen type I and II have been described previously ${ }^{24}$. After an initial denaturation step at $95^{\circ} \mathrm{C}$ for 10 minutes, the cDNA products were amplified with 50 PCR cycles, consisting of a denaturation step at $95^{\circ} \mathrm{C}$ for 15 seconds and an extension step at $60^{\circ} \mathrm{C}$ for 1 minute. Data analysis was carried out using the Sequence Detector $\mathrm{V}$ program (Perkin-Elmer Applied Biosystems). Since Type II collagen is a typical marker of differentiated chondrocytes in hyaline cartilage as opposed to Type I collagen (expressed by dedifferentiated chondrocytes in fibrocartilage), the ratio of mRNA levels of collagen Type II to I $(\mathrm{Cll} / \mathrm{Cl})$ was used as a useful "differentiation index" by which to compare chondrocyte differentiation ${ }^{24}$.

\section{Immunofluorescence analysis of receptors}

At day 19 of culture, cells were fixed for 15 minutes with $4 \%$ paraformaldehyde (Sigma), rinsed with PBS (Invitrogen) and blocked for 30 minutes with serum-free protein block (DAKO, Glostrup, Denmark). Cells were further rinsed with PBS and incubated separately with each of the following monoclonal antibodies: anti- $\alpha 5 \beta 1$ P1D6 (Covance, Princeton NJ), dilution 1:500; anti- $\alpha$ V $\beta 3$ VI-PL2 (Pharmingen, San Diego CA), dilution 1:100; antiCD44/FITC BU52 (Ancell, Bayport MN), dilution 1:100. Cells were washed in PBS and further incubated for 30 minutes with appropriate secondary antibodies (Molecular Probes) except for the pre-conjugated anti-CD44. Cells were then washed 3 times with PBS prior to mounting with an anti-fading medium (Vectashield: Vector Labs, Burlingame CA) and coverslipping. 
Although it is not yet possible to discriminate between occupied and unoccupied integrins visualized by immunofluorescence microscopy, integrin occupancy does not preclude anti$\alpha 5 \beta 1 \mathrm{mAb}$ binding to distinct epitopes on the integrin subunits. The secondary mAb used in these studies is known to inhibit the binding of $\alpha 5 \beta 1$ to a secondary synergy Pro-His-SerArg-Asn (PHSRN) binding sequence in Fn, but does not block the recognition of the RGD motif $^{25,26}$, thereby leaving open the possibility that a population of such integrins may have bound to Fn in a manner that did not include the synergy sequence. Similarly, the BU52 antiCD44 mAb binds to CD44 while allowing concomitant binding to the GAG-rich epitope ${ }^{27,28}$. However, there is little information available regarding anti- $\alpha v \beta 3$ mAb peptide mapping.

\section{Scanning electron microscopy (SEM)}

To examine cell morphology, samples harvested at day 19 were fixed in Karnovsky's fixative and dehydrated by a graded ethanol series and hydroxymethyldisilazane (Sigma). Samples were sputter coated $(10 \mathrm{~nm})$ with gold (Cressington Scientific, Watford, UK) and examined by SEM (Phillips ESEM, Eindhoven, The Netherlands).

\section{Protein adsorption and desorption}

Protein adsorption was studied by immersing 500 $\mu \mathrm{m}$ diameter PEGT:PBT particles of 8 different compositions overnight in $\mathrm{FBS}$ at $37^{\circ} \mathrm{C}$. The particles were then washed three times with PBS to remove passively attached proteins, and boiled in Laemmli buffer (Bio-Rad, Veenendaal, The Netherlands) with 5\% $\beta$-mercaptoethanol (Sigma) (15 minutes) to desorb proteins that had chemisorbed at the surface. Particles were centrifuged and the supernatant $(20 \mu \mathrm{l})$ from each polymer composition normalized to total protein desorbed (RC-DC protein assay: Bio-Rad) were loaded into lanes of SDS-polyacrylamide gels. To test whether all 
proteins had desorbed, the polymer particles were re-boiled in Laemmli buffer and reevaluated for protein content, which was non-detectable.

\section{Western blotting}

Samples were electrophoresed at $120 \mathrm{~V}$. Decasted gels were soaked in blot buffer for 15 minutes and transferred to an Immobilon-P membrane (Millipore, Amsterdam, The Netherlands) by blotting for 1 hour at 60 Volts, $120 \mathrm{~mA}$. Blots were blocked by 30 minutes incubation in PBS / 0.5\% Tween X-100 (Sigma) / 2\% gelatin (CalBiochem, San Diego CA). This was followed by 4 hours incubation at RT with either of the primary antibodies. The primary antibodies used were IST-3 anti-human Fn mAb (Sigma) and anti-bovine Vn pAb (Biotrend, Cologne, Germany). Blots were rinsed $3 \times 10$ minutes in PBS / 0.5\% Tween X-100 followed by incubation for 1 hour at RT in PBS / 0.5\% Tween X-100 / 2\% gelatin plus the appropriate AP-conjugated secondary antibodies (Sigma), followed by rinsing 3 × 10 minutes in PBS / 0.5\% Tween X-100. Detection was performed using a colorimetric AP conjugate substrate kit (Bio-Rad).

\section{Semi-quantitative image analysis}

Stained blots were digitally scanned and relative quantities of $\mathrm{Vn}$ and $\mathrm{Fn}$ determined by automated histogram-based counting of dark versus white pixels, based on specified threshold values. The results are depicted on a scale of arbitrary densitometric units where the amount of dark pixels were normalized to the fixed overall band area.

\section{Cell quantitation}

Quantitation of total DNA was performed by Cyquant Cell Proliferation assay kit (Molecular Probes, Leiden, The Netherlands) using a spectrofluorometer (Perkin Elmer, IL). Cell 
numbers were derived from DNA content by using results which show that there is 10pg DNA per human chondrocyte ${ }^{29}$.

Statistical Analysis

Analysis of significance (2-tailed distribution) was performed to study the correlation between polymer contact angle, cell attachment, $\mathrm{CII} / \mathrm{Cl}$ ratio, Fn and $\mathrm{Vn}$ adsorption (SPSS, Chicago IL). 


\section{RESULTS}

The phenotype-modulating effects of the polymer materials are reported first, followed by an examination of the underlying mechanisms involved in the cellular and molecular response.

\section{Analysis of mRNA transcript expression by real time-PCR}

Quantitative analyses of type II and type I collagen messenger RNA (mRNA) expression in cells cultivated for 19 days revealed the highest collagen II : collagen I (CII/CI) mRNA ratio for chondrocytes cultured on 1000-PEG 70:30 films (CII/Cl=7.3) (Fig. 2a). The lowest $\mathrm{ClI} / \mathrm{Cl}$ mRNA ratios were found on the TCPS controls $(\mathrm{CII} / \mathrm{Cl}=0.065)$. Cells on the 300-PEG 70:30 substrates demonstrated a marginally higher $\mathrm{CII} / \mathrm{Cl}$ mRNA ratio than those on 1000-PEG 40:60, whereas chondrocytes on the remaining $300 \mathrm{~g} /$ mole surfaces had lower $\mathrm{CII} / \mathrm{Cl} \mathrm{mRNA}$ ratios than on any other PEGT:PBT substrate. Within the $1000 \mathrm{~g} / \mathrm{mole}$ PEG group, only 1000-PEG 40:60 (CII/Cl=0.6) had a CII/CI mRNA ratio of less than 1 (Fig. 2a).

Plots of $\mathrm{Cl} / \mathrm{Cl}$ mRNA ratios versus either $\mathrm{Fn}$ or $\mathrm{Vn}$ revealed a significant inverse correlation with Fn adsorption (significant to the 0.05 level (2-tailed)), but no correlation with Vn (Fig. 2b). However, chondrocyte $\mathrm{CII} / \mathrm{Cl}$ mRNA expression ratios plotted against surface contact angles indicated no direct correlation (data not presented).

\section{Receptor expression}

Immunofluorescence microscopy at day 19 revealed the expression of the $\mathrm{Vn}$-receptor $\alpha \mathrm{V} \beta 3$ integrin and of the Fn-receptor $\alpha 5 \beta 1$ integrin at the focal adhesions of chondrocytes cultivated on PEGT:PBT substrates with $300 \mathrm{~g} / \mathrm{mole}$ PEG (Fig. 3a, b). The data presented 
here are from 300-PEG 55:45 substrates and are representative of the $300 \mathrm{~g} / \mathrm{mole}$ PEG polymers. No Fn or Vn-integrin receptors were detected in cells cultured on $1000 \mathrm{~g} / \mathrm{mole}$ polymers at any time point (data not presented).

Immunofluorescence imaging for CD44, a proteoglycan transmembrane receptor, revealed strikingly different results. At day 19, CD44 was seen to be expressed abundantly in chondrocytes cultivated on $1000 \mathrm{~g} /$ mole PEG substrates (Fig. 3c), but its expression was negligible in cells cultured on substrates with $300 \mathrm{~g} / \mathrm{mole}$ PEG (Fig. 3d).

\section{Chondrocyte morphology}

Chondrocytes cultured on 4000-PEG 55:45 polymer substrates and all $1000 \mathrm{~g} / \mathrm{mole}$ PEG substrates were spheroid in shape (Fig. 4a), although spreading increased with polymer PBT content (not shown).

Cell morphology was generally spread on $300 \mathrm{~g} /$ mole PEG materials. Fig. 4b shows an example of the spread, fibroblastic morphology on the composition with the lowest PEGT content $(30 \%)$. Chondrocytes that were adhered to the control TCPS exhibited spread and near-confluent morphologies (not shown).

\section{Protein adsorption}

Semi-quantitative gel analysis of Western blots of fibronectin (Fn) and vitronectin ( $\mathrm{Vn}$ ) adsorbed to polymers of different compositions revealed adsorption trends that depended on polymer composition (Fig. 5a). On the 4000-PEG 55:45 composition, there was 7 times more Vn than Fn. Polymer compositions with $1000 \mathrm{~g} / \mathrm{mole}$ PEG molecular mass generally demonstrated increasing amounts of both $\mathrm{Fn}$ and $\mathrm{Vn}$ as the percentage of PEGT was 
reduced from $70 \%$ to $40 \%$ (i.e. decreasing wettability). However, there was a pronounced increase in Fn across the same range of decreasing wettability, with the relative amount of Fn compared to $\mathrm{Vn}$ increasing from almost $1: 8$ to $1: 1$.

On 300 g/mole PEG substrates, lower Fn but higher Vn adsorption correlated with wettability, as measured by captive bubble techniques on water-equilibrated polymer films. There was always greater adsorption of Fn on the $300 \mathrm{~g} /$ mole PEG polymers than either of the 1000 g/mole or $4000 \mathrm{~g} /$ mole PEG molecular weight ranges (Fig. 5a).

When individual adhesive protein adsorption was plotted against substrate-water contact angle, Fn adsorption exhibited large variability in the $40^{\circ}$ to $50^{\circ}$ contact angle range (Fig. $5 \mathrm{~b}$ ). Vn adsorption remained within a range, regardless of contact angle. There was no significant correlation between $\mathrm{Vn}$ or Fn adsorption and wettability.

\section{Cell attachment}

Chondrocyte numbers were determined by quantitation of DNA in cell lysates one day after seeding (Fig. 6a). The tissue culture polystyrene (TCPS) controls contained the most cells $(17,400$ cells/well). On PEGT:PBT polymer compositions however, the highest cell attachment was seen on 300-PEG 30:70 (9,200 cells/well). Polymers with the same length of PEG chains but with decreasing PEGT content exhibited higher cell numbers. This trend was seen at surfaces of each PEG molecular weight group. As the length of PEG molecules was shortened from $1000 \mathrm{~g} / \mathrm{mole}$ to $300 \mathrm{~g} / \mathrm{mole}$ while PEGT:PBT ratio was held constant, cell attachment displayed an increase. Differences in cell number when PEG chain lengths were

shortened from $4000 \mathrm{~g} / \mathrm{mole}$ to $1000 \mathrm{~g} / \mathrm{mole}$ were less pronounced. Although low at the lowest $\left(10^{\circ}\right)$ and highest $\left(90^{\circ}\right)$ contact angles, chondrocyte attachment increased on some 
substrates with contact angles between $40^{\circ}$ and $54^{\circ}$ (Figure 6b). However, cell attachment remained comparatively low on other substrates within the same general range of wettability, suggesting that wettability was not the sole parameter influencing chondrocyte attachment.

When chondrocyte attachment was examined as a function of protein adsorption, it was seen that the amount of Fn adsorbed directly correlated with cell attachment (correlation was significant at the 0.01 level (2-tailed)) (Fig. 6c). However, no correlation could be made for cell attachment and $\mathrm{Vn}$ adsorption (Fig. 6c). 


\section{DISCUSSION}

There has recently been considerable interest in the development of "smart materials" that are able to regulate the behavior of adhered or encapsulated cells by releasing bioactive molecules into the local environment, or through extracellular protein/peptide mimetics built into the delivery substrates ${ }^{30}, 31$. However, the ability of materials to modulate downstream gene response without exogenous growth factors, coatings or complex ligand incorporation has the potential to greatly facilitate the development of tissue engineering and cellular therapies. As an illustration of this concept, a class of biphasic calcium phosphate ceramic induced de novo bone formation at non-osseous sites in vivo without requiring the delivery of cells or biologic compounds ${ }^{32}$, suggesting that the surface chemistry of the ceramic allowed the selective adsorption of morphogenetic proteins that trigger osteogenesis. It was also demonstrated using polymer libraries that substrate chemistry can influence the developmental lineages of embryonic stem cells ${ }^{33}$. Our data presented here with chondrocytes cultivated on PEGT:PBT substrates compliment these results, demonstrating that certain materials have an intrinsic potential to induce highly specific cell behavior, including modulation of phenotype (Figs. 2, 3, 4).

Our objective for this study was to shed light on the interfacial events that integrate the materials, proteins, cell surface receptors and subsequent downstream gene expression by cells adhered to polymeric delivery substrates. Protein adsorption was shown to correlate with chondrocyte differentiation index and attachment (Figs. 2b, 6c). Interestingly, examination of data as a function of contact angle revealed an independence of protein adsorption and chondrocyte attachment on substrate wettability (Figs. 5, 6). These analyses suggest that differential adhesion protein adsorption to materials, and not simply surface 
wettability, plays a significant role in regulating cell function. It should be noted, however, that since contact angles provide wettabilities that are integrated across the entire analyzed surface area, the actual situation is complex and is likely to be at least partially governed by the Angstrom/nano-scale phase separated structure of the underlying PEGT:PBT surface, as described earlier for this class of polymers ${ }^{34}$.

The pro-chondrogenic potential of specific compositions of poly(ether ester) copolymers may be explained by examining substrate-protein and protein-receptor interactions. PEG molecules in water are in a liquid-like state, with rapid movement and a large excluded volume, with high PEG content surfaces providing the greatest steric interference ${ }^{35}$. This is also applicable to the PEGT:PBT substrates synthesized and studied herein, and was examined using PEG length and weight ratio. Changes in substrate composition and wettability have been shown to influence $\mathrm{Fn}$ conformation $^{36}$, with conformational interplay between competitively adsorbed $\mathrm{Fn}$ and albumin at the material-protein interface enhancing FN activity ${ }^{37}$. The amount of surface PEG in other polymers has been shown to inversely correlate with Fn coating and affects its conformation ${ }^{38}$. The effects of protein folding have not been examined in this study, however, and is an area that needs further study with respect to the biomaterial interface. Both $\mathrm{Vn}$ and $\mathrm{Fn}$ can exist in either folded or extended conformations $^{39}, 40$, binding to cells primarily via integrin binding to Arg-Gly-Asp (RGD) sequence domain $\mathrm{s}^{41,42}$, as well as by glycosaminoglycan (GAG)-binding motifs in the vicinity of the carboxyl terminus that bind to transmembrane proteoglycans ${ }^{43-47}$. Normal differentiated chondrocytes express and bind to their hyaluronic acid-rich extracellular matrix via CD44, a proteoglycan transmembrane receptor that can also bind to the GAG-binding domains of the adsorbed proteins ${ }^{48}$. Therefore, we postulate that the differentiated chondrogenic phenotype 
observed on high density PEG polymers was due to CD44-protein interaction via GAGbinding domains.

Interestingly, it has been previously reported that $\mathrm{Vn}$ adsorption was not inhibited by increasing PEG concentration at a surface, regardless of substrate wettability ${ }^{49}$, and that $\mathrm{Vn}$ adsorbed equally well to untreated PS as to surface modified TCPS ${ }^{50}$. Both studies compliment our data that suggest that substrate wettability alone does not strongly influence $\mathrm{Vn}$ adsorption. Whereas native $\mathrm{Vn}$ does not have strong affinity for GAG, this affinity is greatly increased upon adsorption to surfaces due to the unfolding of the protein and subsequent exposure of GAG-binding domains ${ }^{51}$. Since up to $20 \%$ of $\mathrm{Vn}$ in serum is already in the extended GAG-binding conformation ${ }^{51}$, the likelihood of GAG-binding domains to be exposed to the chondrocyte plasma membrane is significant, and would be in addition to any binding domain exposure by adsorption to the hydrophobic PBT domains of the polymer substrate.

Chondrocytes that exhibited higher chondrogenic gene expression (per the differentiation index) also maintained a spheroid morphology (Figs. 3, 4), whereas Fn adsorption resulted in chondrocyte dedifferentiation by the formation of focal adhesion plaques containing the $\alpha 5 \beta 1$ integrin receptor (Fig. 3). The relationship between cell shape and differentiation has been widely described $^{13,14,52}$, and we have previously shown that causing a change in cell shape by inhibiting RhoA activation enabled the reversion of the chondrogenic phenotype from a dedifferentiated state ${ }^{53}$. It was also recently reported that causing mesenchymal stem cells to conform to either a spread or spheroid shape differentially induced cell differentiation towards the osteogenic and adipogenic lineages, respectively, due to shape-driven influences in signaling pathways ${ }^{54}$. Chondrocytes from healthy human articular cartilage are known to 
express $\alpha 5 \beta 1$ integrin, whereas $\alpha v \beta 3$ is only weakly expressed ${ }^{55}$. However, in vitro monolayer culture on TCPS markedly increased surface expression of these integrins ${ }^{56}$ and resulted in chondrocyte dedifferentiation. Since spheroid chondrocytes in vitro also show greater incorporation of ${ }^{35} \mathrm{SO}_{4}$ into GAG than spread chondrocytes ${ }^{57}$, the adsorption of $\mathrm{Fn}$ on substrates may also play a role in cell spreading, in addition to adhesion, and is a phenomenon not found in healthy cartilage in vivo.

In conclusion, this study suggests a strong role of material modification in creating biomimetic and cellular effects that, until now, were principally expected from biological moieties such as growth factors. This was illustrated by modulation of the chondrogenic phenotype through differential induction of cell-surface receptors that coincided with different quantities of substrate-bound Fn or Vn. These events influence cell shape and downstream gene expression, and can be engineered by changing polymer composition to provide the molecular cues required for individual therapeutic applications.

\section{ACKNOWLEDGEMENTS}

We are grateful to Drs. M. Humphries and J. Bezemer for helpful discussions regarding this manuscript. 


\section{REFERENCES}

1. Langer, R. \& Vacanti, J. Tissue engineering. Science 260, 920-926 (1993).

2. Tabata, Y. Tissue regeneration based on growth factor release. Tissue Eng 9 Suppl 1, S5-15 (2003).

3. Chen, R.R. \& Mooney, D.J. Polymeric growth factor delivery strategies for tissue engineering. Pharm Res 20, 1103-1112 (2003).

4. Stoker, M., O'Neill, C., Berryman, S. \& Waxman, V. Anchorage and growth regulation in normal and virus-transformed cells. Int J Cancer 3, 638 (1968).

5. Maroudas, N.G. Sulphonated polystyrene as an optimal substratum for the adhesion and spreading of mesenchymal cells in monovalent and divalent saline solutions. J Cell Physiol 90, 511 (1977).

6. Curtis, A., Forrester, J., McInnes, C. \& Lawrie, F. Adhesion of cells to polystyrene surfaces. J Cell Biol 97, 1500-1506 (1983).

7. Cima, L. et al. Tissue Engineering by cell transplantation using degradable polymer substrates. J Biomech Eng 113, 143-151 (1991).

8. Woodfield, T.B., Bezemer, J.M., Pieper, J.S., van Blitterswijk, C.A. \& Riesle, J. Scaffolds for tissue engineering of cartilage. Crit Rev Eukaryot Gene Expr 12, 209-236 (2002).

9. Hunziker, E.B. Articular cartilage repair: basic science and clinical progress. A review of the current status and prospects. Osteoarthritis Cartilage 10, 432-463 (2002).

10. Saltzman, W.M. in Principles of Tissue Engineering, Edn. 2. (eds. R.P. Lanza, R. Langer \& J. Vacanti) 221-235 (Academic Press, San Diego; 2000).

11. Horbett, T. \& Schway, M. Correlations between mouse $3 T 3$ cell spreading and serum fibronectin adsorption on glass and hydroxyethylmethacrylate-ethylmethacrylate copolymers. $J$ Biomed Mater Res 22, 763-793 (1988).

12. Lee, M.H., Ducheyne, P., Lynch, L., Boettiger, D. \& Composto, R.J. Effect of biomaterial surface properties on fibronectin-alpha5beta1 integrin interaction and cellular attachment. Biomaterials 27, 1907-1916 (2006).

13. Loty, S., Forest, N., Boulekbache, H. \& Sautier, J.M. Cytochalasin D induces changes in cell shape and promotes in vitro chondrogenesis: a morphological study. Biol Cell 83, 149-161 (1995).

14. Martin, I., Vunjak-Novakovic, G., Yang, J., Langer, R. \& Freed, L.E. Mammalian chondrocytes expanded in the presence of fibroblast growth factor 2 maintain the ability to differentiate and regenerate three-dimensional cartilaginous tissue. Exp Cell Res 253, 681-688 (1999). 
15. Martin, I. et al. Enhanced cartilage tissue engineering by sequential exposure of chondrocytes to FGF-2 during 2D expansion and BMP-2 during 3D cultivation. J Cell Biochem 83, 121-128 (2001).

16. Benya, P.D. \& Shaffer, J.D. Dedifferentiated chondrocytes reexpress the differentiated collagen phenotype when cultured in agarose gels. Cell 30, 215-224 (1982).

17. Wang, L. et al. Flow cytometric analysis of the human articular chondrocyte phenotype in vitro. Osteoarthritis Cartilage 9, 73-84 (2001).

18. Pennypacker, J.P., Hassell, J.R., Yamada, K.M. \& Pratt, R.M. The influence of an adhesive cell surface protein on chondrogenic expression in vitro. Exp Cell Res 121, 411-415 (1979).

19. West, C.M. et al. Fibronectin alters the phenotypic properties of cultured chick embryo chondroblasts. Cell 17, 491-501 (1979).

20. Wyre, R.M. \& Downes, S. The role of protein adsorption on chondrocyte adhesion to a heterocyclic methacrylate polymer system. Biomaterials 23, 357-364 (2002).

21. Deschamps, A.A., Grijpma, D.W. \& Feijen, J. Poly(ethylene oxide)/poly(butylene terephthalate) segmented block copolymers: the effect of copolymer composition on physical properties and degradation behavior. Polymer 42, 9335-9345 (2001).

22. Papadaki, M. et al. The different behaviors of skeletal muscle cells and chondrocytes on PEGT/PBT block copolymers are related to the surface properties of the substrate. J Biomed Mater Res 54, 47-58 (2001).

23. Deschamps, A.A. et al. Design of segmented poly(ether ester) materials and structures for the tissue engineering of bone. J Control Release 78, 175-186 (2002).

24. Martin, I. et al. Quantitative analysis of gene expression in human articular cartilage from normal and osteoarthritic joints. Osteoarthritis Cartilage 9, 112-118 (2001).

25. Mould, A.P. et al. Defining the topology of integrin $\alpha 5 \beta 1$-fibronectin interactions using inhibitory anti- $\alpha 5$ and anti- $\beta 1$ monoclonal antibodies. J Biol Chem 272, 17283-17292 (1997).

26. Burrows, L., Clark, K., Mould, A.P. \& Humphries, M.J. Fine mapping of inhibitory anti- $\alpha 5$ monoclonal antibody epitopes that differentially affect integrin-ligand binding. Biochem $J \mathbf{3 4 4}$, 527-533 (1999).

27. Okamoto, I. et al. Regulated CD44 cleavage under the control of protein kinase C, calcium influx, and the Rho family of small G proteins. J Biol Chem 274, 25525-25534 (1999).

28. Anstee, D. et al. New monoclonal antibodies in CD44 and CD58: their use to quantify CD44 and CD58 on normal human erythrocytes and to compare the distribution of CD44 and CD58 in human tissues. Immunology 74, 197-205 (1991).

29. Jakob, M. et al. Enzymatic digestion of adult human articular cartilage yields a small fraction of the total available cells. Connect Tissue Res 44, 173-180 (2003).

30. Anderson, D.G., Burdick, J.A. \& Langer, R. Materials science. Smart biomaterials. Science 305, 1923-1924 (2004). 
31. Lutolf, M.P. \& Hubbell, J.A. Synthetic biomaterials as instructive extracellular microenvironments for morphogenesis in tissue engineering. Nat Biotechnol 23, 47-55 (2005).

32. Yuan, H.P. et al. A comparison of the osteoinductive potential of two calcium phosphate ceramics implanted intramuscularly in goats. Journal of Materials Science-Materials in Medicine 13, 1271-1275 (2002).

33. Anderson, D.G., Levenberg, S. \& Langer, R. Nanoliter-scale synthesis of arrayed biomaterials and application to human embryonic stem cells. Nat Biotechnol 22, 863-866 (2004).

34. Dechamps, A.A., Grijpma, D.W. \& Feijen, J. Poly(ethylene oxide)/poly(butylene terephthalate) segmented block copolymers: the effect of copolymer composition on physical properties and degradation behavior. Polymer 42, 9335-9345 (2001).

35. Lee, J.H., Kopecek, J. \& Andrade, J.D. Protein-resistant surfaces prepared by PEO-containing block copolymer surfactants. J Biomed Mater Res 23, 351-368 (1989).

36. Altankov, G. et al. Modulating the biocompatibility of polymer surfaces with poly(ethylene glycol): effect of fibronectin. J Biomed Mater Res 52, 219-230 (2000).

37. Grinnell, F. Fibronectin adsorption on material surfaces. Ann N Y Acad Sci 516, 280-290 (1987).

38. Tziampazis, E., Kohn, J. \& Moghe, P.V. PEG-variant biomaterials as selectively adhesive protein templates: model surfaces for controlled cell adhesion and migration. Biomaterials 21, 511-520 (2000).

39. Erickson, H.P. \& Carrell, N.A. Fibronectin in extended and compact conformations. Electron microscopy and sedimentation analysis. J Biol Chem 258, 14539-14544 (1983).

40. Williams, E.C., Janmey, P.A., Ferry, J.D. \& Mosher, D.F. Conformational states of fibronectin. Effects of $\mathrm{pH}$, ionic strength, and collagen binding. J Biol Chem 257, 14973-14978 (1982).

41. Ruoslahti, E. \& Pierschbacher, M.D. Arg-Gly-Asp: a versatile cell recognition signal. Cell 44, 517-518 (1986).

42. Suzuki, S., Oldberg, A., Hayman, E.G., Pierschbacher, M.D. \& Ruoslahti, E. Complete amino acid sequence of human vitronectin deduced from cDNA. Similarity of cell attachment sites in vitronectin and fibronectin. Embo J 4, 2519-2524 (1985).

43. Dalton, B.A., McFarland, C.D., Underwood, P.A. \& Steele, J.G. Role of the heparin binding domain of fibronectin in attachment and spreading of human bone-derived cells. J Cell Sci 108 (Pt 5), 2083-2092 (1995).

44. Liang, O.D., Rosenblatt, S., Chhatwal, G.S. \& Preissner, K.T. Identification of novel heparinbinding domains of vitronectin. FEBS Lett 407, 169-172 (1997).

45. Woods, A., McCarthy, J.B., Furcht, L.T. \& Couchman, J.R. A synthetic peptide from the $\mathrm{COOH}$-terminal heparin-binding domain of fibronectin promotes focal adhesion formation. Mol Biol Cell 4, 605-613 (1993).

46. Schvartz, I., Seger, D. \& Shaltiel, S. Vitronectin. Int J Biochem Cell Bio/ 31, 539-544 (1999). 
47. Suzuki, S. et al. Domain structure of vitronectin. Alignment of active sites. J Biol Chem 259, 15307-15314 (1984).

48. Knudson, W. \& Loeser, R.F. CD44 and integrin matrix receptors participate in cartilage homeostasis. Cell Mol Life Sci 59, 36-44 (2002).

49. Fabrizius-Homan, D.J. \& Cooper, S.L. Competitive adsorption of vitronectin with albumin, fibrinogen, and fibronectin on polymeric biomaterials. J Biomed Mater Res 25, 953-971 (1991).

50. Pitt, W.G., Fabrizius-Homan, D.J., Mosher, D.F. \& Cooper, S.L. Vitronectin adsorption onto polystyrene and oxidized polystyrene. J Colloid Interface Sci 129, 231-239 (1989).

51. Preissner, K.T. Structure and biological role of vitronectin. Annu Rev Cell Biol 7, 275-310 (1991).

52. Loty, C., Forest, N., Boulekbache, H., Kokubo, T. \& Sautier, J.M. Behavior of fetal rat chondrocytes cultured on a bioactive glass-ceramic. J Biomed Mater Res 37, 137-149 (1997).

53. Mahmood, T.A., de Jong, R., Riesle, J., Langer, R. \& van Blitterswijk, C.A. Adhesion-mediated signal transduction in human articular chondrocytes: the influence of biomaterial chemistry and tenascin-C. Exp Cell Res 301, 179-188 (2004).

54. McBeath, R., Pirone, D.M., Nelson, C.M., Bhadriraju, K. \& Chen, C.S. Cell shape, cytoskeletal tension, and RhoA regulate stem cell lineage commitment. Dev Cell 6, 483-495 (2004).

55. Loeser, R.F. Chondrocyte integrin expression and function. Biorheology 37, 109-116 (2000).

56. Loeser, R.F., Carlson, C.S. \& McGee, M.P. Expression of beta 1 integrins by cultured articular chondrocytes and in osteoarthritic cartilage. Exp Cell Res 217, 248-257 (1995).

57. Glowacki, J., Trepman, E. \& Folkman, J. Cell shape and phenotypic expression in chondrocytes. Proc Soc Exp Biol Med 172, 93-98 (1983). 


\begin{tabular}{lll}
\hline $\begin{array}{l}\text { Polymer composition, per applied } \\
\text { nomenclature }\end{array}$ & PEG molecular mass & PEGT:PBT mass ratio \\
\hline 4000-PEG 55:45 & 4000 & \\
\hline 1000-PEG 70:30 & 1000 & $55: 45$ \\
\hline 1000-PEG 55:45 & 1000 & $70: 30$ \\
\hline 1000-PEG 40:60 & 1000 & $55: 45$ \\
\hline 300-PEG 70:30 & 300 & $40: 60$ \\
\hline 300-PEG 55:45 & 300 & $70: 30$ \\
\hline 300-PEG 30:70 & 300 & $55: 45$ \\
\hline TCPS (tissue culture polystyrene) & n/a & $30: 70$ \\
\hline
\end{tabular}

TABLE 1 


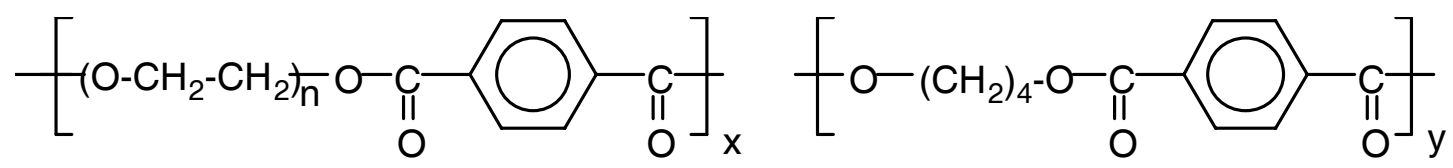

poly(ethylene glycol) terephthalate

poly(butylene terephthalate)

\section{FIGURE 1}




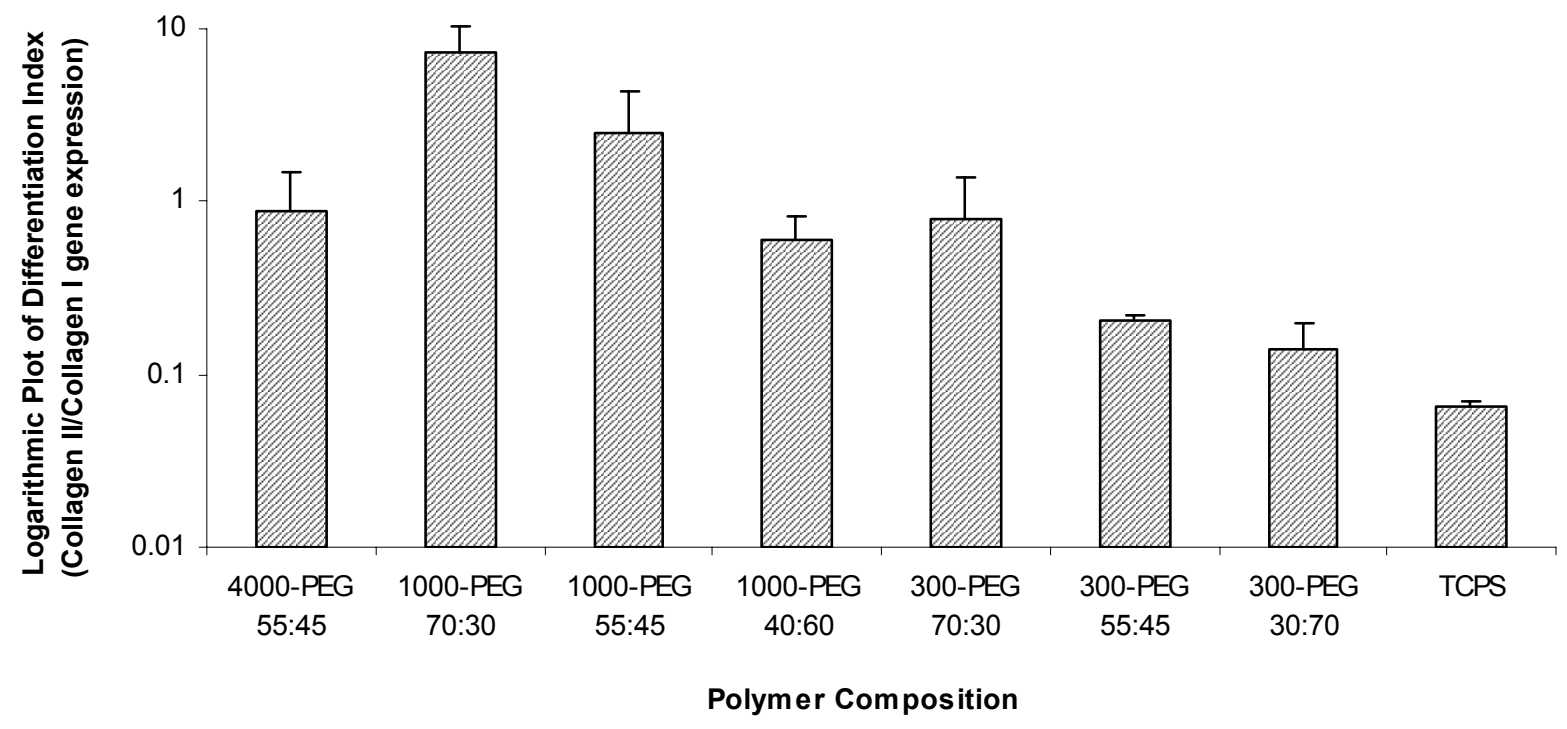

FIGURE 2a

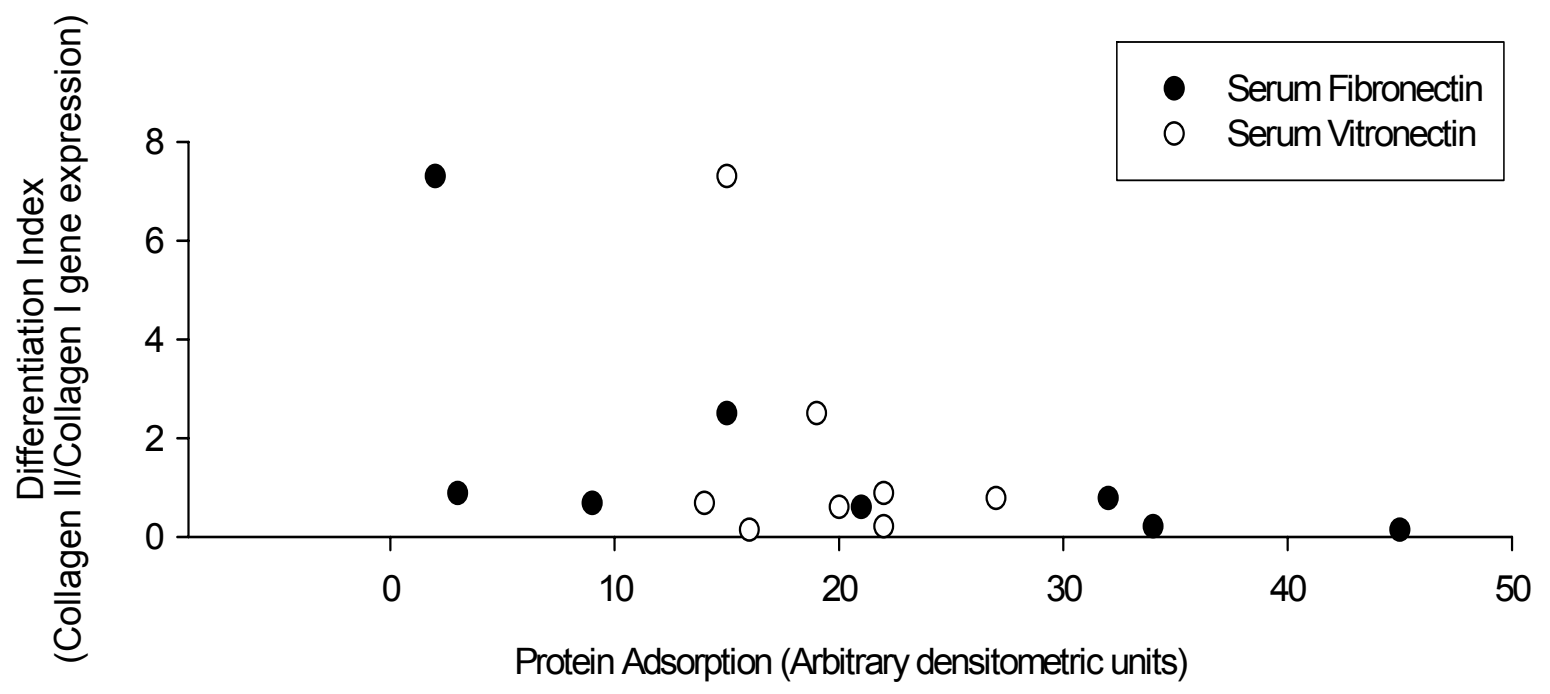

FIGURE 2b 


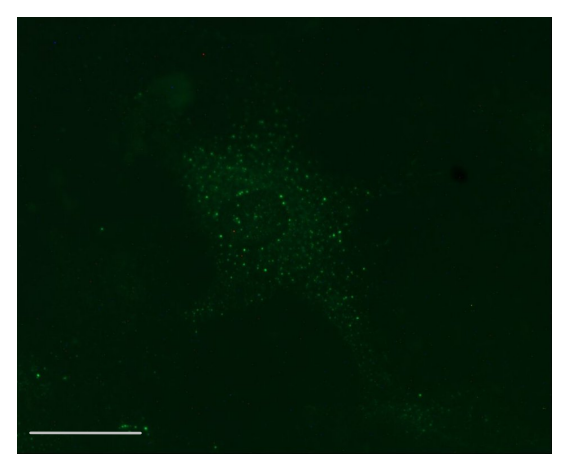

FIGURE 3a

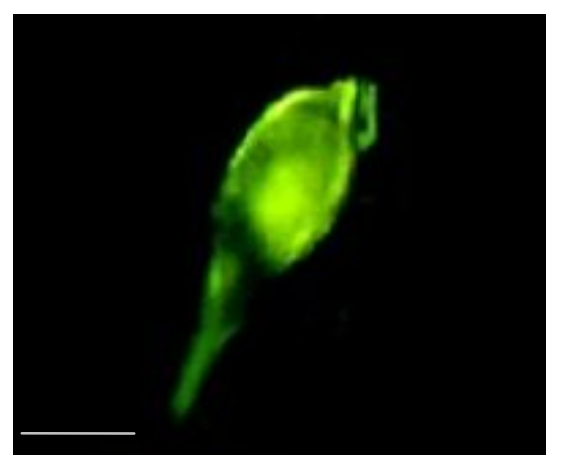

FIGURE 3c

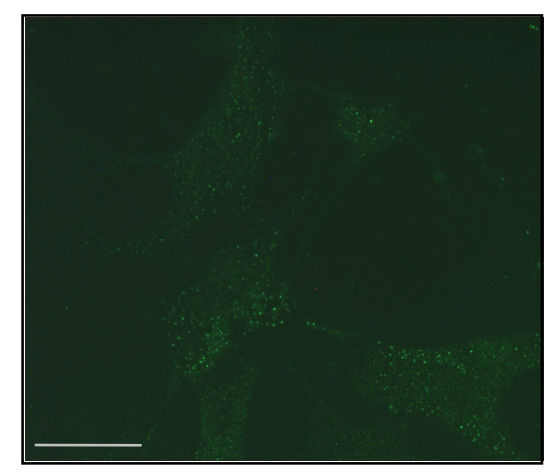

FIGURE 3b

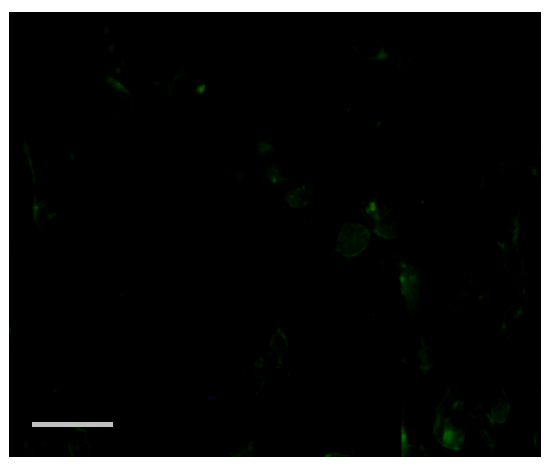

FIGURE 3d 


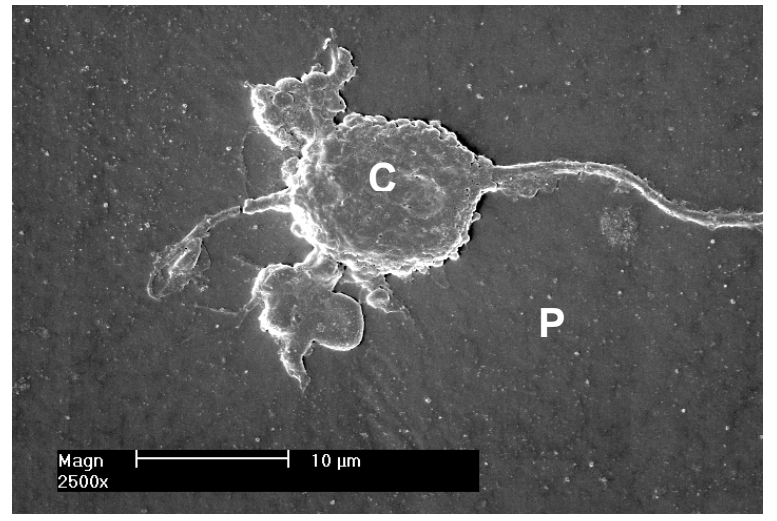

FIGURE 4a

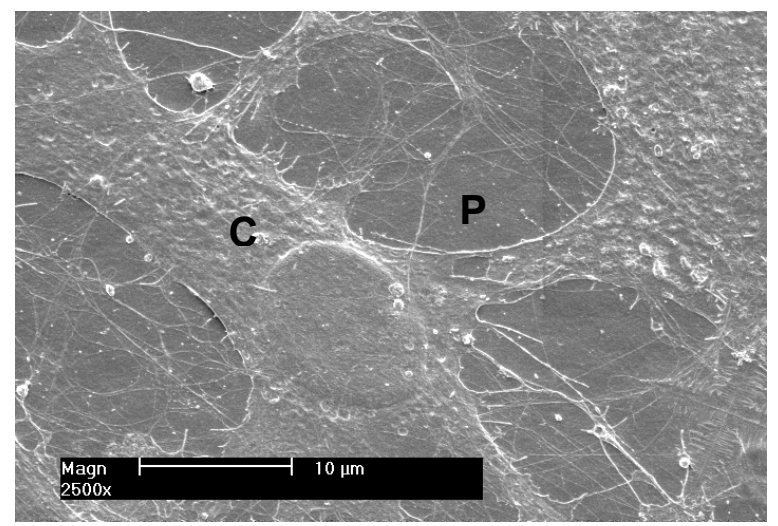

FIGURE 4b 


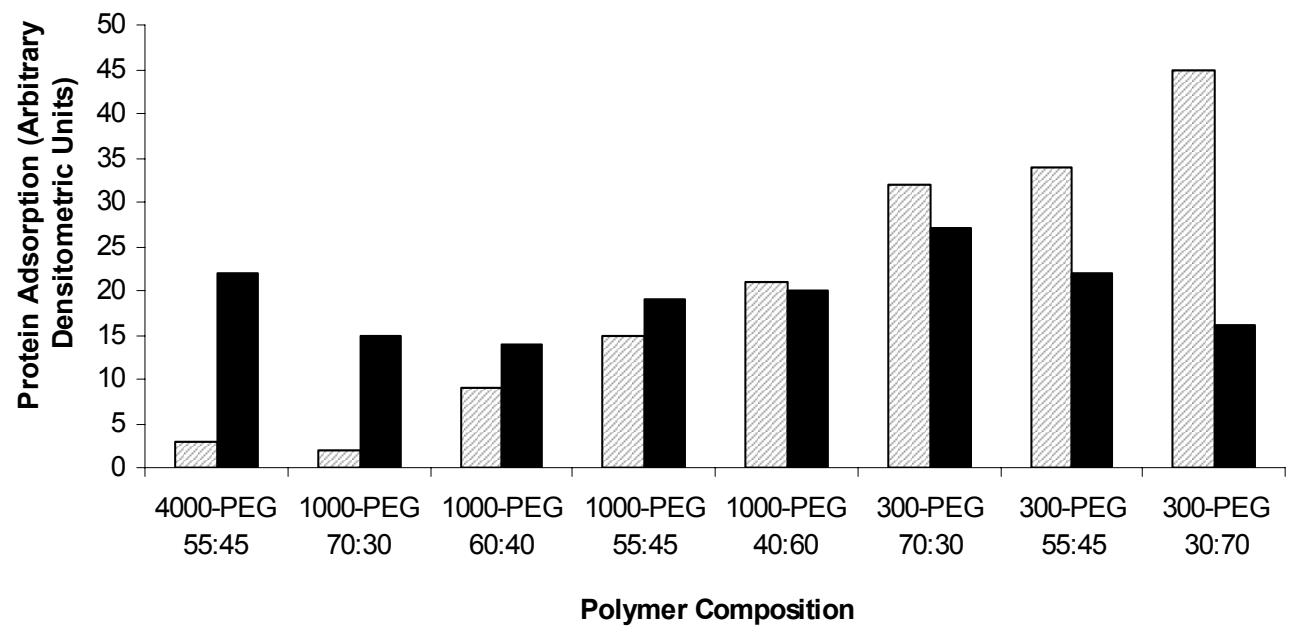

FIGURE 5a

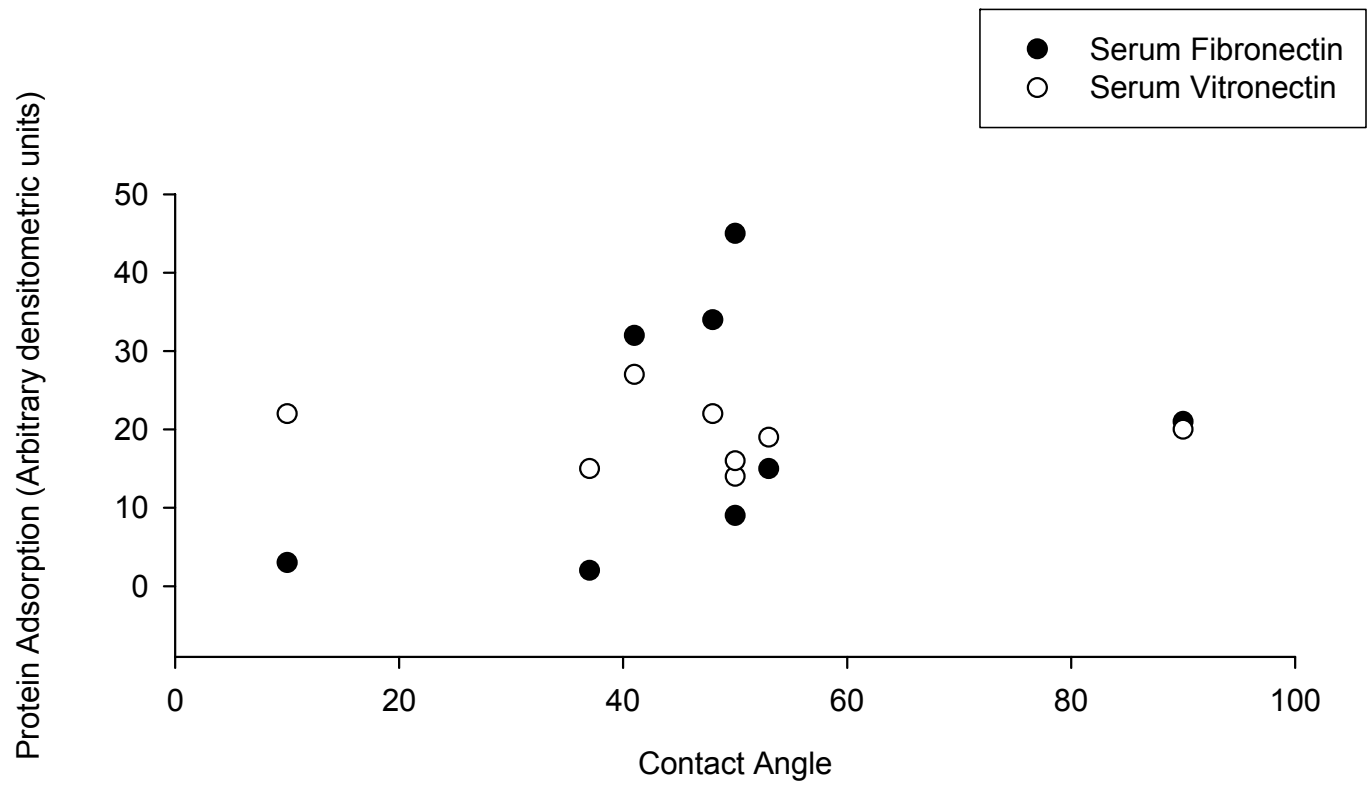

FIGURE 5b 


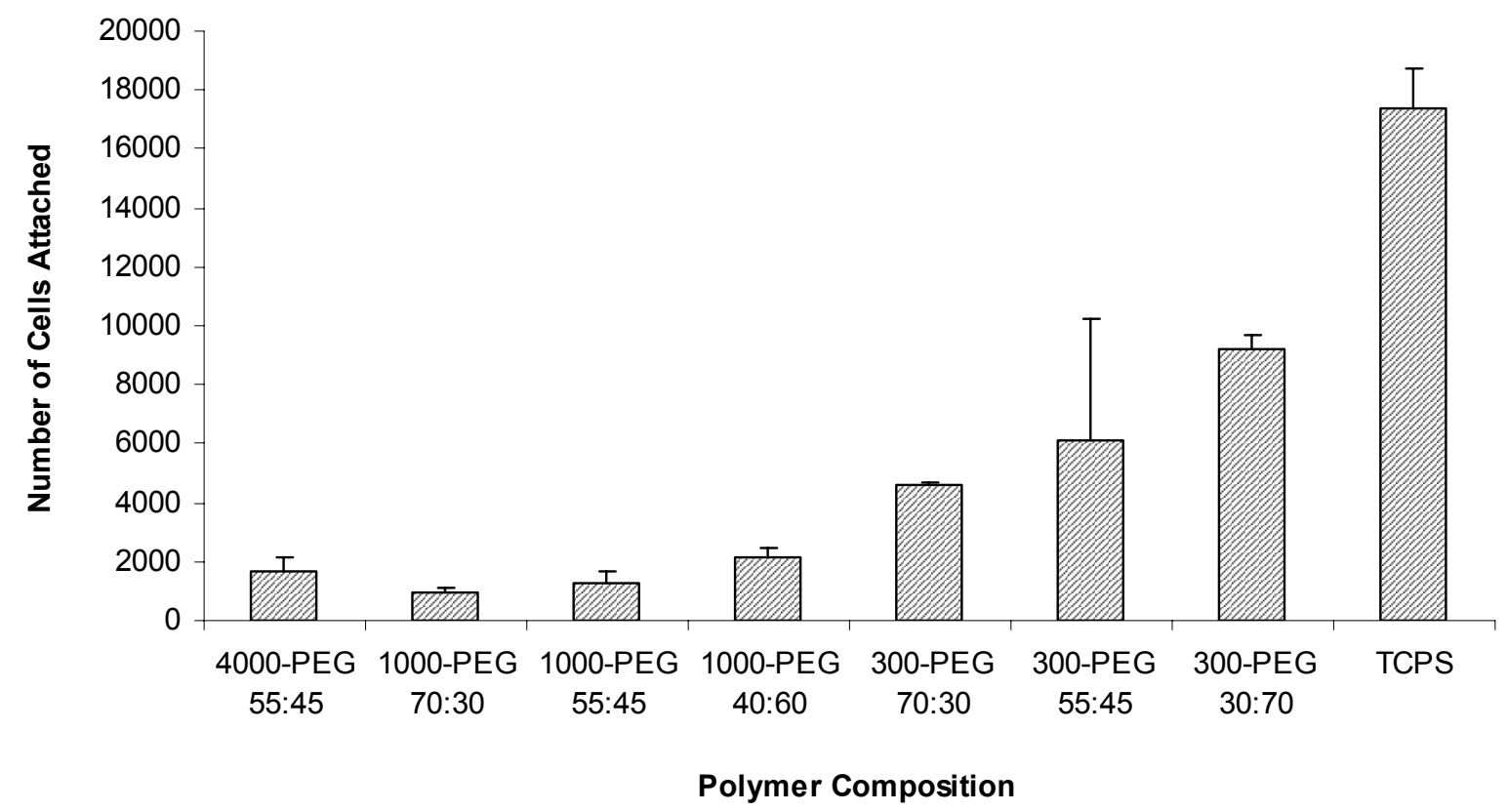

FIGURE 6a

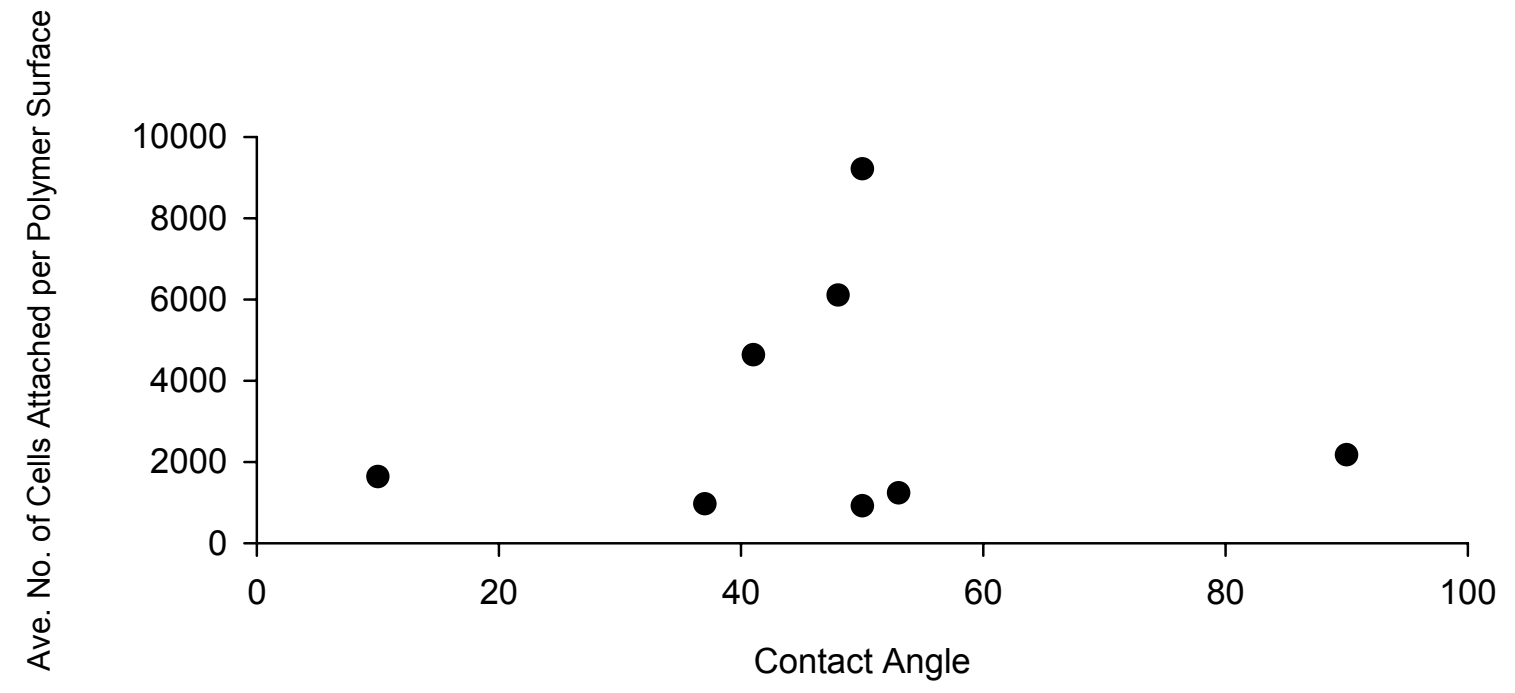

FIGURE 6b 


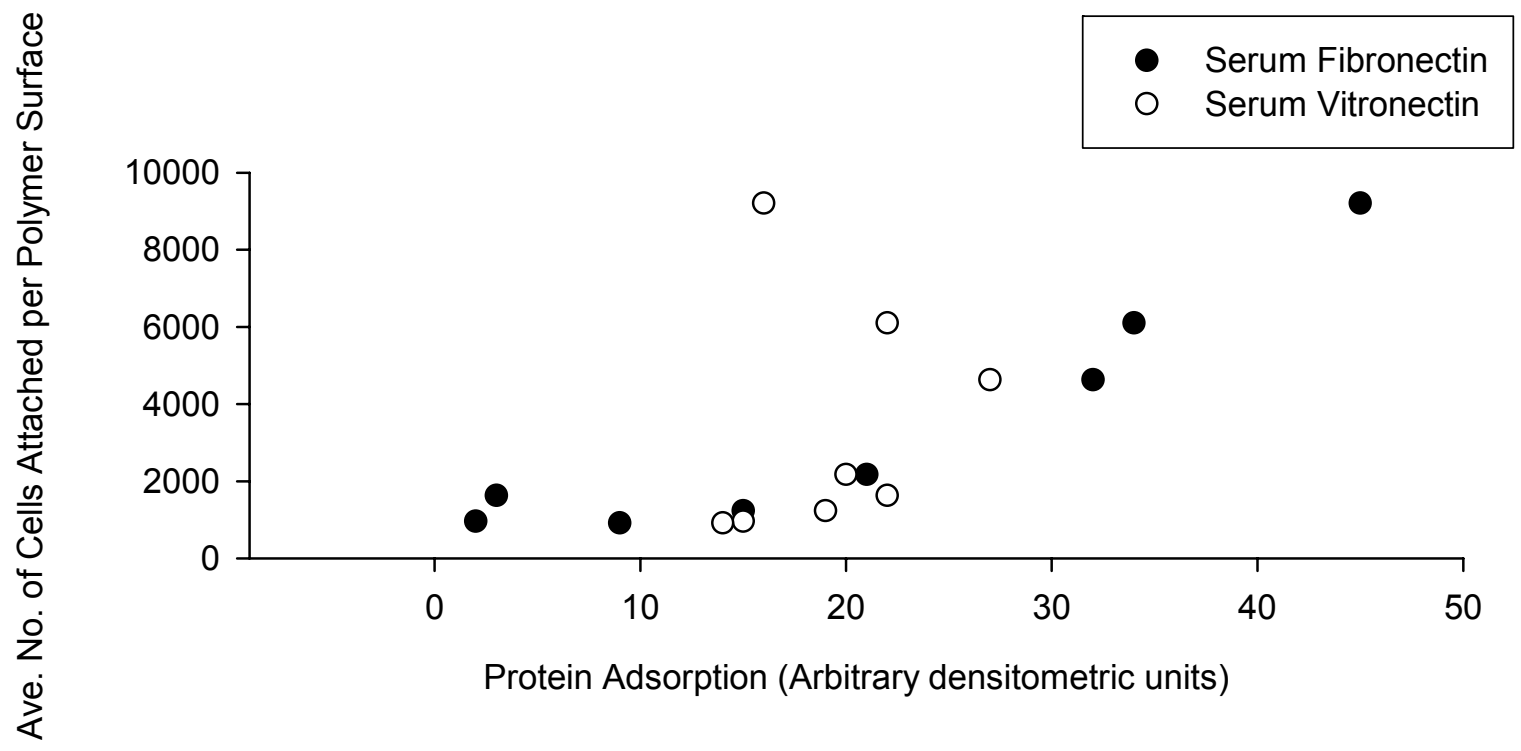

FIGURE 6C 


\section{TABLE AND FIGURE LEGENDS}

Table 1.

A list of compositions of the model PEGT:PBT segmented copolymer system, per the nomenclature used in this study.

Figure 1.

Chemical structure of segmented poly(ether ester) [PEGT:PBT] copolymers, formed by polycondensation polymerization of hydrophilic PEG-containing segments and hydrophobic PBT segments.

Figure 2.

Chondrocyte $\mathrm{CII} / \mathrm{Cl}$ mRNA expression on substrates with varying PEG molecular mass and PEGT:PBT ratio (Fig. 2a), and $\mathrm{CII/CI}$ mRNA expression compared against $\mathrm{Fn}$ and $\mathrm{Vn}$ adsorption (Fig. 2b). The correlation between Fn adsorption and dedifferentiation was significant. In Fig. 2a, polymers compositions are arranged from left by PEG molecular mass of 4000,1000 and $300 \mathrm{~g} / \mathrm{mole}$.

Figure 3.

Immunofluorescence images of the $\mathrm{Vn}$ receptor $\alpha \mathrm{v} \beta 3$ (Fig. 3a), Fn receptor $\alpha 5 \beta 1$ (Fig. 3b) expressed by chondrocytes cultivated on 300-PEG 55:45 substrates, as well as the proteoglycan receptor CD44 expressed by chondrocytes cultivated on 1000-PEG 70:30 (Fig. 3c) and on 300-PEG 55:45 (Fig. 3d) at day 19. Scale bars: Fig. 3a - $20 \mu \mathrm{m}$; Fig. $3 \mathrm{~b}-40 \mu \mathrm{m}$; 


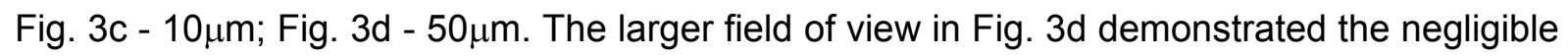
expression of CD44 by chondrocytes cultivated on 300-PEG 55:45.

Figure 4.

SEM images of a rounded chondrocyte on 1000-PEG 70:30 (Fig. 4a) and chondrocytes with spread morphologies on 300-PEG 30:70 (Fig. 4b), both at 19 days post-seeding. Cells and polymer substrate are identified by the labels " $C$ " and "P", respectively.

Figure 5.

Relative adsorption of $\mathrm{Vn}$ and Fn on polymer substrates with varying PEG molecular mass and PEGT:PBT ratio (Fig. 5a). The bars correspond to Fn (clear) and Vn (solid). Polymers are arranged from left by PEG molecular mass of 4000, 1000 and $300 \mathrm{~g} / \mathrm{mole}$. Fig. $5 \mathrm{~b}$ relates the adsorption of Fn and $\mathrm{Vn}$ to substrate wettability.

Figure 6.

Average number of cells at day 1 attached to substrates with varying PEG molecular mass and PEGT:PBT ratio (Fig. 6a). Polymers are arranged from left by PEG molecular mass of 4000, 1000 and $300 \mathrm{~g} / \mathrm{mole}$. Substrate wettability versus cell number is plotted in Fig. 6b. The relationship between cell number at day 1 and protein adsorption is demonstrated in Fig. $6 \mathrm{c}$, where the correlation between cell attachment and Fn adsorption was significant. 